\title{
Neutrophil involvement in Covid-19
}

\author{
Athanasios Didangelos (PhD) \\ ad482@leicester.ac.uk
}

\section{University of Leicester, Mayer IgA Nephropathy Laboratory, University of Leicester, Leicester, LE1 7RH, United Kingdom}

\begin{abstract}
Covid-19 is often related to hyperinflammation that drives lung or multi-organ injury. The immunopathological mechanisms that cause excessive inflammation following SARS-Cov-2 infection are under investigation while different approaches to limit hyperinflammation in affected patients are being proposed. Here, a computational protein-protein interaction network approach was used on recently available data to identify possible Covid-19 inflammatory mechanisms and bioactive genes. First, network analysis of putative SARS-Cov-2 cellular receptors and their directly associated proteins, led to the mining of a robust neutrophil response signature and multiple relevant inflammatory genes. Second, analysis of RNA-seq datasets of lung epithelial cells infected with SARS-Cov-2 revealed that infected cells specifically expressed neutrophil-attracting chemokines, further supporting the likely role of neutrophils in Covid-19 inflammation. Third, analysis of RNA-seq datasets of bronchoalveolar lavage fluid from Covid-19 patients, identified neutrophil-specific genes and chemokines. Different immunoregulatory and neutrophil-relevant molecules mined here such as, TNFR, IL8, CXCR1, CXCR2, ADAM10, GPR84, MME-neprilysin, ANPEP and LAP3 are druggable and might be therapeutic targets in efforts to limit SARS-Cov-2 inflammation in severe clinical cases. The role of neutrophils in Covid-19 needs to be studied further.
\end{abstract}

\section{Introduction}

New studies have highlighted that Covid-19 is often characterised by an extreme inflammatory response associated with lung and multi-organ injury and mortality and have suggested promising anti-inflammatory options (1). Other studies recommend caution with immunosuppression given that regulated inflammation is necessary for an effective anti-viral response (2). More ideas are needed to understand Covid-19 hyperinflammation and target relevant immunopathological mechanisms. The role and function of different myeloid cell types in Covid-19 lung injury, cytokine storm and sepsis are not well understood. This short study is focused on mining inflammatory signatures using simple protein-protein interaction networks of coronavirus receptors and recently published RNA-seq datasets on SARS-Cov-2 infected human cells.

\section{Methods}

The data used here for protein analysis was collected from published sources as cited. Lung epithelial cell RNA-seq data was downloaded from Blanco-Melo et al., 2020 (Supplemental Table 2) (3). Bronchoalveolar lavage fluid (BALF) RNA-seq dataset was reproduced from Xiong et al., 2020 (Supplementary Table 1) (4). Network analysis was performed using StringDB. For the 7 coronavirus receptors, network inflation was performed on StringDB(v10) by adding 100 first-shell (direct firstdegree protein interactions). For lung epithelial cell and BALF RNA-seq datasets only significantly upregulated genes (same criteria as in the studies) were used to construct protein-protein interaction networks. All network interactions were recorded and used as edges (0.150 - 0.999). Gene Ontology analysis was performed using GeneOntology and validated in BiNGO in Cytoscape. Drug-Gene interactions were downloaded from DGldb. Networks, network analysis, comparisons and final designs were built on Cytoscape.

\section{Results and Discussion}

A recent study highlighted 7 putative SARS-Cov-2 receptors including the now well-known ACE2 (5) together with peptidases DPP4 and ANPEP as well as pathogen-binding proteins CD209, CLEC4G, CLEC4M and CEACAM1 (Fig.1A). These proteins are expressed in different cell types and are implicated in the binding of coronaviruses on epithelial cells (6). To examine these proteins further, the small signature (Fig.1A) was inflated in StringDB to construct a protein-protein interaction network 
with up to 100 proteins that directly interact with the 7 input proteins (Fig.1B). Network inflation was performed to identify proteins that directly associate with the putative coronavirus receptors on human cells, thereby allowing mining of possible functional pathways. Surprisingly, the main ontology of this expanded network was "Neutrophil Degranulation" (GO:0043312) with 70 proteins in this category (Fig.1C). There were also 8 neutrophil-enriched genes (Neutrophil-enriched genes) (7) including ANPEP, MME, MGAM, CD177, CEACAM1/3, FPR2 and CYSTM1 (Fig.1D). Thus, coronavirus binding proteins (and their direct interactors) might be involved in neutrophil or other related classic inflammatory mechanisms. Two neutrophil genes, ANPEP and CEACAM1 (Fig.1D) are coronavirus receptors (Fig.1A) indicating that neutrophils could be infected by SARS-Cov-2, as they are by Influenza A (8). At the time of writing this paper, interaction or infection of neutrophils with SARS-Cov2 had not been reported.

The role of neutrophils in viral infections of the upper respiratory tract and their possible involvement in therapeutic strategies is not entirely clarified (9). They are involved in early anti-viral defence (10) but through degranulation and lysis, they can also be cytotoxic during ARDS and severe pneumonia, including from coronaviruses (11). Neutrophil hyperinflammation is also likely in other severe viral infections such as Hepatitis (9). In current Covid-19 literature, increased peripheral neutrophil-tolymphocyte ratio is observed in severe cases (12) but not much is known regarding neutrophils in Covid-19 affected lungs. Covid-19 lung injury in specific patients might involve dysregulated neutrophil activity.

To examine neutrophil recruitment from the perspective of Covid-19, I analysed a published RNA-seq dataset of human lung epithelial cells infected with SARS-Cov-2 (3), to find gene expression changes following SARS-Cov-2 infection. Regulated genes are presented in (Fig.1E). As expected, the gene signature contains 39 highly connected inflammation and viral-response genes, including classic inflammatory mediators and interferon pathway genes (Fig.1F). Notably, the SARS-Cov-2 infected lung epithelial cells overexpressed 6 chemokines that belong to the human ontology annotation "Neutrophil Chemotaxis" (GO:0030593), and include the classic neutrophil chemoattractants CXCL1, CXCL2, CXCL3, CXCL5, IL8 (CXCL8) and CCL20 (Fig.1G), suggesting that these cells can express neutrophil chemokines after SARS-Cov-2 infection. The receptors for these chemokines (CXCR2 and CXCR1; IL8 receptor) are neutrophil-enriched genes like CXCL1, CXCL5, IL8, ANPEP and CEACAM1 (Neutrophil-enriched genes). SARS-Cov-2 infected lung cells also overexpressed complement $\mathrm{C} 3$ and associated pathway activation genes $(\mathrm{Fig} .1 \mathrm{H})$, while the receptor for the $\mathrm{C} 3 \mathrm{a}$ anaphylatoxin (C3AR1) is a "Neutrophil Degranulation" gene found by network inflation of the 7 putative SARS-Cov-2 receptors (Fig.1B). C3 and complement activation have been recently involved in ARDS with systemic inflammation and lung neutrophilia (13).

Finally, analysis of a published RNA-seq dataset of human bronchoalveolar lavage fluid (BALF) from just 2 Covid-19 patients (4), revealed the upregulation of 18 in total (Fig.1I) neutrophil-enriched genes (PPL, ENCUR, STEAP4, SLP1, MUC21, HEY1, MUC21, CXCL1) and neutrophil chemotaxis genes (CXCX2, CCL2, CXCL6, CCL8, CCL2, TGFB2, CCL3L3, CCL4L4), further supporting likely involvement of neutrophils in Covid-19 lungs.

Are neutrophils and related inflammatory mechanisms likely targets in Covid-19 complications? This is a difficult question given the complexity of acute innate immune responses and the importance of neutrophils in early anti-viral defence. Universal suppression of neutrophils or other myeloid cell types is not trivial and the clinical evidence on the use of steroids in Covid-19 inconclusive. Nevertheless, it might be possible to target specific inflammatory mechanisms before ARDS. The target-mining presented here includes multiple druggable proteins (Fig.1J-K) such as neutrophil-attracting chemokine signalling (Fig.1J), and neutrophil-relevant inflammatory entities (Fig.1J), as well as SARS-Cov-2 receptors (Fig.1K).

IL8 (Fig.1J), a cardinal neutrophil chemoattractant and product of activated neutrophils, can be blocked by neutralising antibodies (HuMax-IL8). The neutrophil chemokine receptors CXCR1 and CXCR2 are targeted by different experimental drugs including the CXCR2 blocker AZD5069 (Fig.1J). 
Other interesting inflammatory proteins include the monocyte and neutrophil genes TNF-receptor-2 (TNFR2 or TNFRSF1B), GPR84 and ADAM10 (Fig.1J). TNFR2 is clinically blocked by the receptorantagonist Enbrel. While TNF has a well-established role in neutrophil activation and prolongs neutrophil survival (14), the role of TNF in Covid-19 infection has not received attention. GPR84 and ADAM 10 are targeted with experimental drugs including the trialled GPR84 blocker GLPG-1205 and the ADAM10 inhibitor llomastat (Fig.1J). FPR2 (formyl peptide receptor), MME (neprilysin) and MGAM (maltase-glucoamylase) (Fig.1J) are directly involved in inflammatory neutrophil recruitment and activity in many diseases.

ANPEP (Aminopeptidase-N), a likely coronavirus receptor (Fig.1A) and a neutrophil-enriched gene (Fig.1D), is potentially blocked by approved drugs Ezetimibe and Icatibant, while there is experimental evidence that it interacts with Tosedostat and Ubenimex. It is however unlikely that binding of these drugs to the aminopeptidase will interfere with viral binding. Ubenimex is also an inhibitor of LAP3 (leucine aminopeptidase-3), the only gene shared between the coronavirus receptors inflated network (Fig.1B) and SARS-Cov-2 infected lung epithelial cells (Fig.1F). LAP3 was induced in lung epithelial cells following SARS-Cov-2 infection and it is a monocyte and neutrophil-enriched gene. Like other aminopeptidases (i.e. ANPEP), LAP3 might act as a coronavirus receptor.

\section{Conclusions}

Neutrophil recruitment and related inflammatory activity might be important components of severe Covid-19 immunopathology. In contrast to universal immunosuppression, specific inflammatory proteins and pathways could be considered in patients suffering from Covid-19 hyperinflammation. Moreover, the role of neutrophils in recognition of SARS-Cov-2 and the possibility that they are infected with the virus needs to be studied further. Finally, it is perhaps worth noting that some currently investigated Covid-19 therapeutic options include hydroxychloroquine, azithromycin and colchicine. All three have well-studied anti-neutrophil effects (15-17).

\section{References}

1. Mehta P, McAuley DF, Brown M, Sanchez E, Tattersall RS, Manson JJ, HIh Across Speciality Collaboration UK. 2020. COVID-19: consider cytokine storm syndromes and immunosuppression. Lancet 395:1033-1034.

2. Ritchie Al, Singanayagam A. 2020. Immunosuppression for hyperinflammation in COVID-19: a double-edged sword? Lancet 395:1111.

3. Blanco-Melo D, Nilsson-Payant BE, Liu W-C, Møller R, Panis M, Sachs D, Albrecht RA, tenOever BR. 2020. SARS-CoV-2 launches a unique transcriptional signature from in vitro, ex vivo, and in vivo systems. bioRxiv doi:10.1101/2020.03.24.004655:2020.03.24.004655.

4. Xiong Y, Liu Y, Cao L, Wang D, Guo M, Jiang A, Guo D, Hu W, Yang J, Tang Z, Wu H, Lin Y, Zhang M, Zhang Q, Shi M, Liu Y, Zhou Y, Lan K, Chen Y. 2020. Transcriptomic characteristics of bronchoalveolar lavage fluid and peripheral blood mononuclear cells in COVID-19 patients. Emerg Microbes Infect 9:761-770.

5. Ou X, Liu Y, Lei X, Li P, Mi D, Ren L, Guo L, Guo R, Chen T, Hu J, Xiang Z, Mu Z, Chen X, Chen J, Hu K, Jin Q, Wang J, Qian Z. 2020. Characterization of spike glycoprotein of SARSCoV-2 on virus entry and its immune cross-reactivity with SARS-CoV. Nat Commun 11:1620.

6. Qi F, Qian S, Zhang S, Zhang Z. 2020. Single cell RNA sequencing of 13 human tissues identify cell types and receptors of human coronaviruses. Biochem Biophys Res Commun doi:10.1016/j.bbrc.2020.03.044.

7. Uhlen M, Karlsson MJ, Zhong W, Tebani A, Pou C, Mikes J, Lakshmikanth T, Forsstrom B, Edfors F, Odeberg J, Mardinoglu A, Zhang C, von Feilitzen K, Mulder J, Sjostedt E, Hober A, Oksvold P, Zwahlen M, Ponten F, Lindskog C, Sivertsson A, Fagerberg L, Brodin P. 2019. A genome-wide transcriptomic analysis of protein-coding genes in human blood cells. Science 366.

8. Hufford MM, Richardson G, Zhou H, Manicassamy B, Garcia-Sastre A, Enelow RI, Braciale TJ. 2012. Influenza-infected neutrophils within the infected lungs act as antigen presenting cells for anti-viral CD8(+) T cells. PLoS One 7:e46581. 
9. Drescher B, Bai F. 2013. Neutrophil in viral infections, friend or foe? Virus Res 171:1-7.

10. Camp JV, Jonsson CB. 2017. A Role for Neutrophils in Viral Respiratory Disease. Front Immunol 8:550.

11. Haick AK, Rzepka JP, Brandon E, Balemba OB, Miura TA. 2014. Neutrophils are needed for an effective immune response against pulmonary rat coronavirus infection, but also contribute to pathology. J Gen Virol 95:578-590.

12. Zheng M, Gao Y, Wang G, Song G, Liu S, Sun D, Xu Y, Tian Z. 2020. Functional exhaustion of antiviral lymphocytes in COVID-19 patients. Cell Mol Immunol doi:10.1038/s41423-0200402-2.

13. Gralinski LE, Sheahan TP, Morrison TE, Menachery VD, Jensen K, Leist SR, Whitmore A, Heise MT, Baric RS. 2018. Complement Activation Contributes to Severe Acute Respiratory Syndrome Coronavirus Pathogenesis. mBio 9.

14. Colotta F, Re F, Polentarutti N, Sozzani S, Mantovani A. 1992. Modulation of granulocyte survival and programmed cell death by cytokines and bacterial products. Blood 80:2012-20.

15. Cronstein BN, Molad Y, Reibman J, Balakhane E, Levin RI, Weissmann G. 1995. Colchicine alters the quantitative and qualitative display of selectins on endothelial cells and neutrophils. J Clin Invest 96:994-1002.

16. Culic O, Erakovic V, Cepelak I, Barisic K, Brajsa K, Ferencic Z, Galovic R, Glojnaric I, Manojlovic Z, Munic V, Novak-Mircetic R, Pavicic-Beljak V, Sucic M, Veljaca M, Zanic-Grubisic T, Parnham MJ. 2002. Azithromycin modulates neutrophil function and circulating inflammatory mediators in healthy human subjects. Eur J Pharmacol 450:277-289.

17. Hurst NP, French JK, Gorjatschko L, Betts WH. 1988. Chloroquine and hydroxychloroquine inhibit multiple sites in metabolic pathways leading to neutrophil superoxide release. J Rheumatol 15:23-7.

\section{Figure Legend}

Fig.1: A: ACE2 and 6 related putative SARS-Cov-2 receptors on epithelial cells. ACE2, DPP4 and ANPEP are peptidases. B-D: The 7 putative SARS-Cov-2 receptors were inflated in StringDB(v10) by adding up to 100 directly interacting proteins with definite association (B). The main gene ontology of the inflated network is "Neutrophil Degranulation" (C) and included 8 Neutrophil-specific genes (D). E-H: 113 genes that were differentially regulated (RNA-seq) in lung epithelial cells following stimulation with SARS-Cov-2 (E). Following virus infection, most upregulated genes were related to inflammatory and virus responses (F). 6 classic neutrophil chemokines were upregulated (G). SARSCov-2 infected epithelial cells also upregulated C3 and related complement pathway genes (H). I: 18 neutrophil-enriched and neutrophil chemotaxis genes that were upregulated (RNA-seq) in BALF collected from 2 Covid-19 patients. J-K: Putative druggable targets with neutrophil and inflammatory function derived from the analysis. Approved and experimental drugs with solid pharmacological evidence are presented as interaction networks. Protein-Drug associations were examined in DGiDB and were manually curated to exclude false-positive or non-experimentally validated interactions. All protein-protein interaction networks were performed in Cytoscape using interaction data derived from StringDB(v10). 


\section{Figure 1}

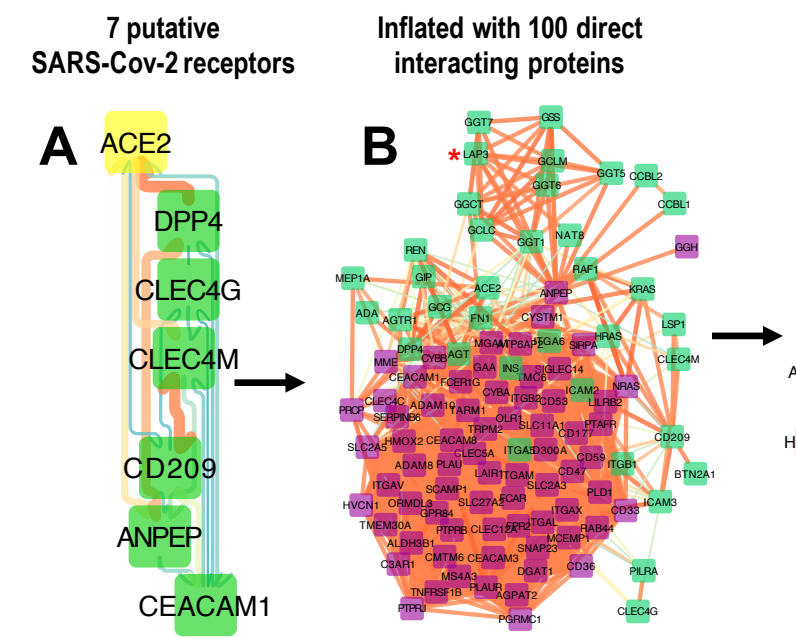

Main Gene Ontology (GO):
Neutrophil Degranulation
8 Neutrophil-enriched genes

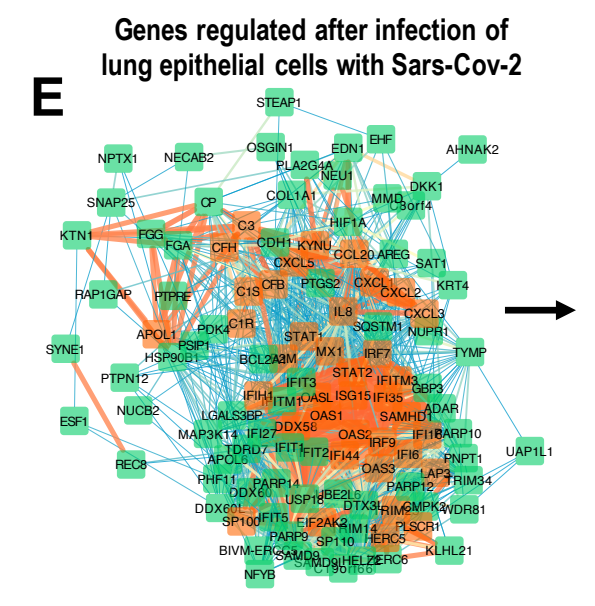

Neutrophil-specific genes upregulated in BALF

$$
\text { I }
$$
KIAA1324 HIST1H2BC MUC21
HIST1H4B STEAP4
PPL CXCL1 ENKUR CCL2 TGFB2

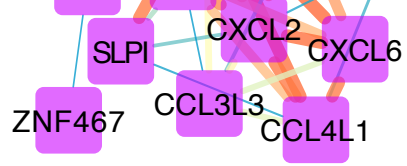

J
Main GO of upregulated genes:

$\mathbf{F}$ Inflammatory \& Virus response LAP3

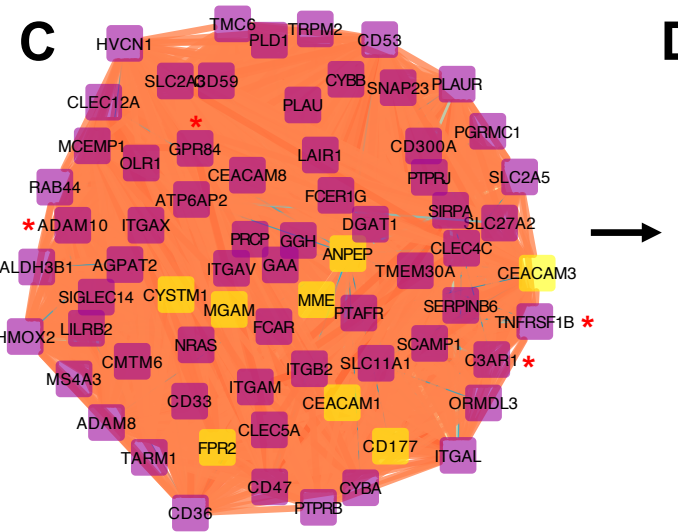

chemoattractants

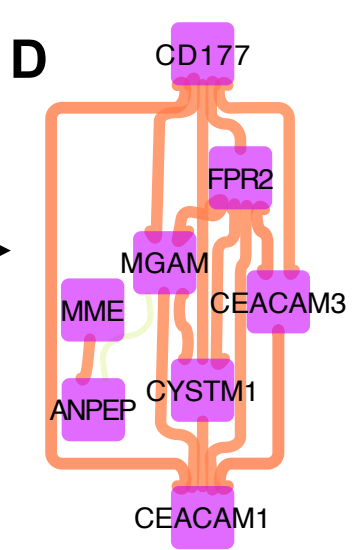

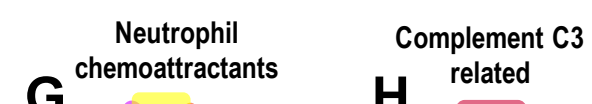

H related

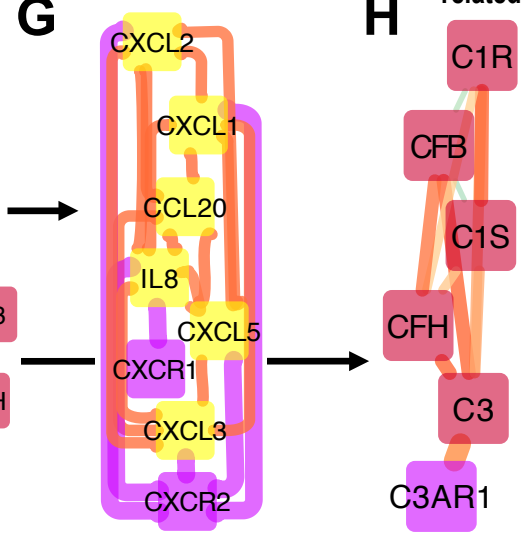

Druggable inflammatory genes PLSCR1 OAS2 OAS1 STAT1 CXCL5 EIF2AK2 DDX58IFIH1 IL8 CCL2O
HERC5 SP100 IFIG IFI35 MX1 IRF7 TRIM25 IFI35 STAT2 TRIM25 SAMHD1 B2M C1S CFH IFITM3 APOL1 1 C1R $\begin{array}{ccc}\text { ENBREL STAVUDINE } & \text { CARTEOLOL } & \begin{array}{c}\text { Putative Sars-Cov-2 } \\ \text { receptor drugs }\end{array} \\ \text { TNFRSF1B } & \text { MME } & \text { TOSEDOSTAT }\end{array}$ TOSEDOSTAT DIDANOSINE SACUBITRIL WRW4 FPR2 PBP10 UBENIMEX ICATIBANT
EZETIMIBE ANPEP AZD5069 RIVANICLINE ELUBRIXIN

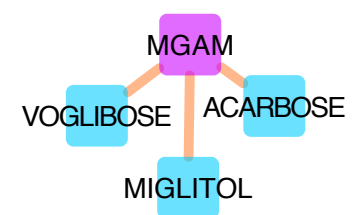
ADAM10 ADERBASIB ILOMASTAT PBI-4050 GLPG-1205 GPR84

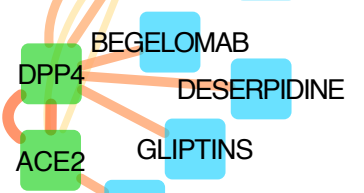

LISINOPRIL 